\title{
THE LASER SWITCHED LINAC AND DEVELOPMENT OF A HIGH BRILLIANC' ELECTRON SOURCE
}

\author{
A.C. Melissinos, C. Bamber, T. Blalock, A. Fry and T. Wilson
}

\section{Department of Physics and Astronomy}

University of Rochester, Rochester, NY 14627

\author{
September 1991
}

\section{DISCLAIMER}

This report was prepared as an account of work sponsored by an agency of the United States Government. Neither the United States Government nor any agency thereof, nor any of their Government. Neither the United States Government or assumes any legal liability or responsiemployees, makes any warranty, express or implied, of any information, apparatus, product, or bility for the accuracy, completeness, or usefulness of any information, apparated proted infringe privately owned rights. process disclosed, or represents that its use would not infringe privately owned trade name, trademark, ence herein to any specific commercial product, process, or or imply its endorsement, recommanufacturer, or otherwise does not necessarily constitute or imply its end thereof. The views mendation, or favoring by the United States Government or an state or reflect those of the and opinions of authors expressed herein do not

\section{MASTER}

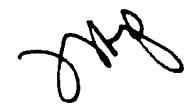

DISTRIBUTION OF THIS DOCUMENT IS UNLIMITED

$$
\begin{aligned}
& \text { Contract wo } \\
& \text { DE-ACO2-76ER } 13065
\end{aligned}
$$




\section{i. Overview of the Program}

This task originated in 1987 to explore the possibility of accelerating short bursts of electrons by pulsed power. The project was originally funded by the AFOSR (Sept. 1987 to Dec. 1990) and since 1988 by the Advanced Technology R\&D Branch of the DHEP, under the present contract. The termination of the AFOSR grant makes it necessary to request this year an incremental funding in this task in order to maintain the activities of the group. A supplemental equipment request is included with this proposal as well.

The principal effort of our group was to demonstrate that electrons can be accelerated by picosecond-long electrical pulses which are compressed in a radial transmission line. This goal has now been achieved and our results are presented in detail in section (ii). We have achieved a gradient of $45 \mathrm{MV} / \mathrm{m}$ across a 250 "m accelerating gap and have accelerated $10^{6}$ electrons in a 1 ps long pulse. The beam emerges from a $500 \mu m$ hole and can be refocused to this transverse dimension. The efficiency of the system, defined as the energy imparted to the electrons divided by the energy in the laser pulse, is of order $\eta=2 \times 10^{-6}$ due to the small number of electrons accelerated. If we identify the gap spacing with one half wavelength of the "accelerating r.f.", our device is equivalent to a $600 \mathrm{GHz}$ structure.

The principal limitation in the accelerating gradient comes from the H.V. hold-off properties of the semiconductor disks that are used as photoconductive switches. We believe that with better materials a factor of 10 can be gained in the gradient. Similarly, the electron yield can be increased by at least three orders of magnitude if proper photocathodes are used in plaie of the metalic surface. (The present photoemission efficiency is $\epsilon=4 \times 10^{-7}$ ). The more difficult problem is the engineering of a multicell structure using our present design of the single cell. Our plans for the continuation of this work are given in section ii (d).

One of the most promising applications of laser switched acceleration is in the operation of a very low emittance electron source. Thus we have turned our attention to this subject, and in particular to building a high brilliance electron source using a superconducting cavity. This project which is being carried out in collaboration with Professor L. Hand and Dr. H. Padamsee of Cornell University is discussed in section (iii). We have 
constructed a cryostat to investigate photoemission from superconducting $\mathrm{Nb}$ and the effect of the laser pulse heating on the superconducting properties of the $\mathrm{Nb}$. We expect to achieve a beam in excess of $5 \mathrm{MeV}$ with a normalized emitance of less than $10^{-6} \mathrm{~m}$-rad. This source would provide a very compact injector for linacs, in particular in free electron laser applications. In our own program we would use the source to convert the electron beam into a high brilliance, 10 Angstrom, X-ray source by backscattering the laser beam off the $5 \mathrm{MeV}$ electrons. This possibility is discussed in section (iv).

Finally we mention that our group has trained several $\mathrm{Ph} . \mathrm{D}$. graduates who are now in the accelerator field. Dr. C. Reece is with the superconducting r.f. group at CEBAF. Dr. J. Rogers is with the NSLS at Brookhaven working on beam instrumentation and on the commissioning of the ATF. Dr. W. Wuensch is a CERN fellow responsible for the construction and testing of the accelerating structures for CLIC. Mr. C. Bamber who will complete his $\mathrm{Ph}$.D. degree this summer will remain with our group continuing research on advanced accelerator technology.

\section{ii. The Laser Switched Linac Project}

As discussed above, this project is nearing completion. Therefore the presentation in this section includes the highlights of the technical goals that were achieved during the course of the project. In particular we discuss (a) Laser development, (b) Electric pulse compression and (c) Electron acceleration. Significant effort was also expended in solid state photoconductive switching where important results have been obtained. These results are documented in the thesis of Dr. L. Kingsley who graduated in January and have been referred to in previous reports of this contract; because of lack of space they will not be repeated here.

\section{(a) Laser Development}

We have built a laser system capable of delivering $30 \mathrm{~mJ}$ pulses of infrared at $\lambda=$ $1.05 \mu \mathrm{m}$. The pulses are compressed to a length of less than $2 \mathrm{ps}$. The overall system is shown schematically in Fig. 1.

The pulses are initially produced in a mode locked Nd:YLF oscillator. The oscillator operates at $100 \mathrm{MHz}$ frequency with pulse length of $60 \mathrm{ps}$ and a few nanojoule of energy in each pulse. The oscillator pulses are focussed into a $7 \mu \mathrm{m}$ diameter optical fiber, $600 \mathrm{~m}$ long. Self-phase modulation in the fiber broadens the spectrum from $2 \dot{A}$ to $20 \dot{A}$. Frequency dispersion over the length of the fiber results in chirped pulses of 140 ps length. Out of this train, one pulse is selected every $400 \mathrm{~ms}$ ( $2.5 \mathrm{~Hz}$ rate) via a Pockels cell (PC) and injected into a regenerative $\mathrm{Nd}$ :glass amplifier. After about 100 passes the injected pulse is amplified up to $3 \mathrm{~mJ}$ and extracted by switching the PC to half-voltage. 


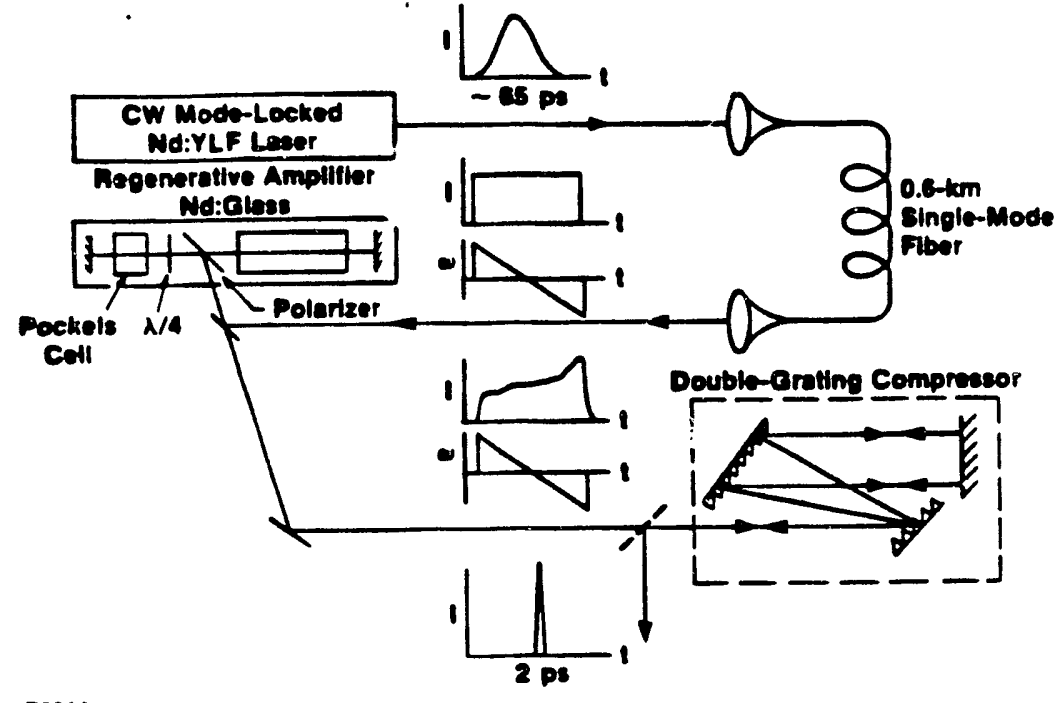

Es14
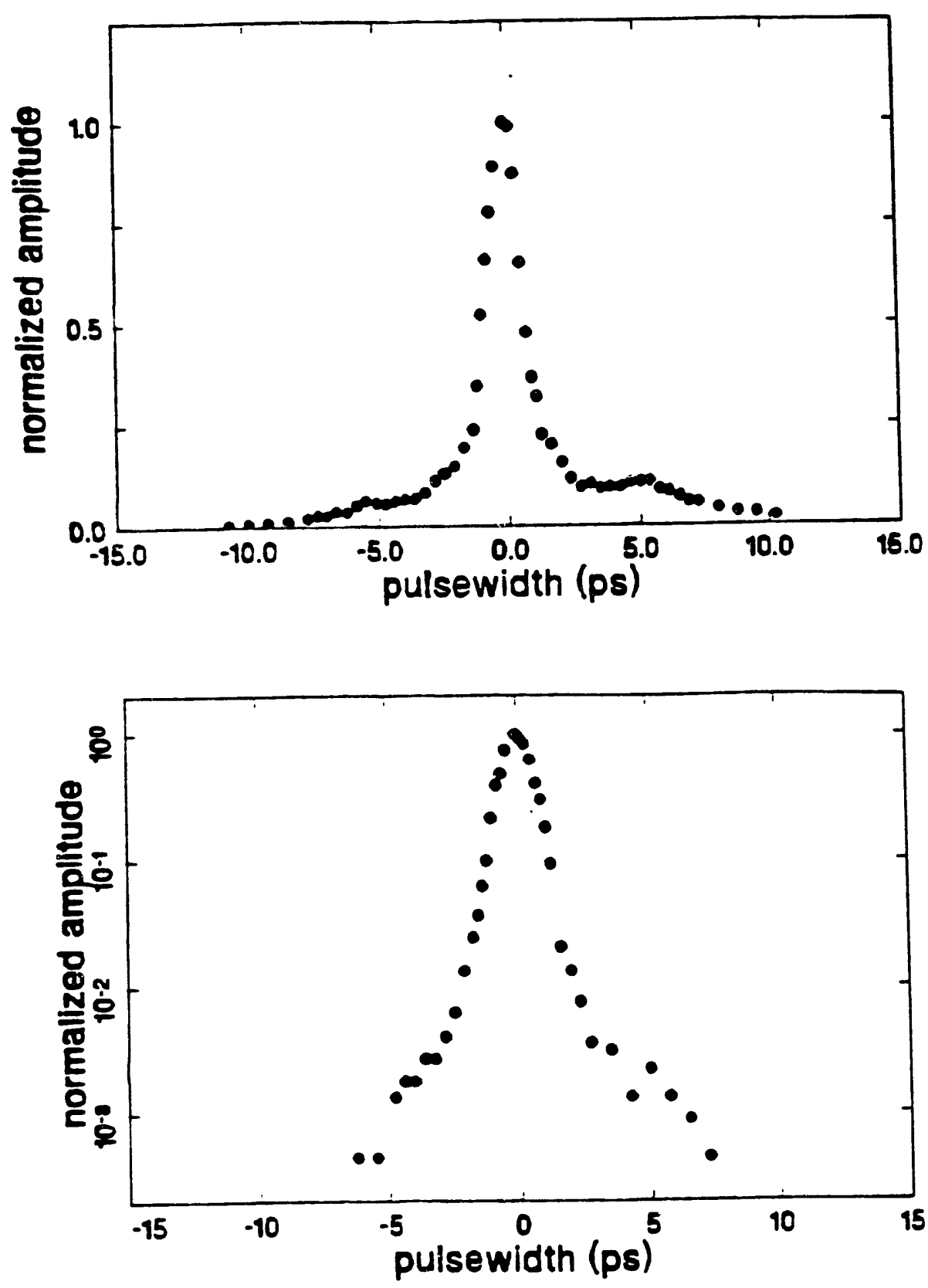

Figure 1: Chirped pulse amplification and compression. A pulse from the mode-locked oscillator is chirped in a fiber and amplified before being compressed to a pulselength of less than 2 psec.

Figure 2(a): Autocorrelation of the compressed pulse. Assuming a gaussian profile, this measurement corresponds to a pulsewidth of 1.7 picoseconds. Almost half of the energy is in the pedestal.

Figure 2(b): Autocorrelation of pulse after saturable absorber. Non-linear absorption attenuates the pedestal much more than the main pulse. The pulsewidth is also sheitened to 1.3 picosecond. 


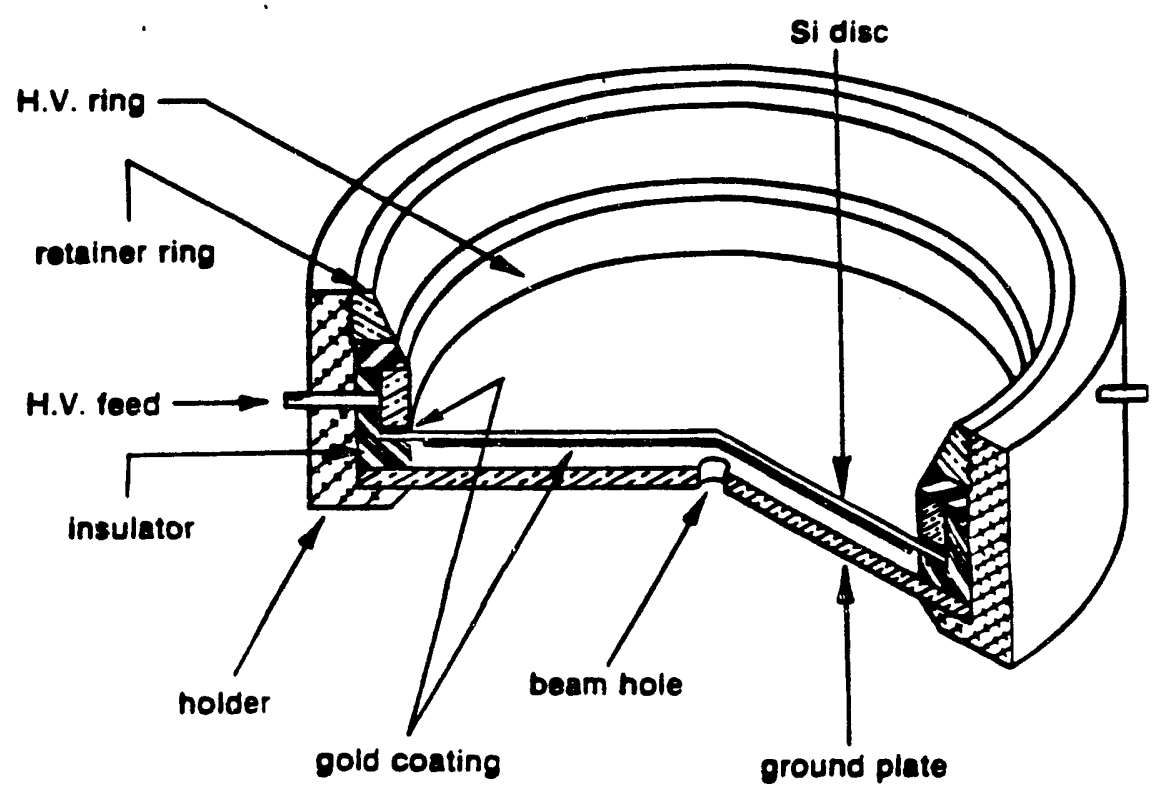

Figure 3: Accelerating disc structure. This radial tranmission line has a radius of $3.1 \mathrm{~cm}$ and a gap of $0.5 \mathrm{~mm}$.

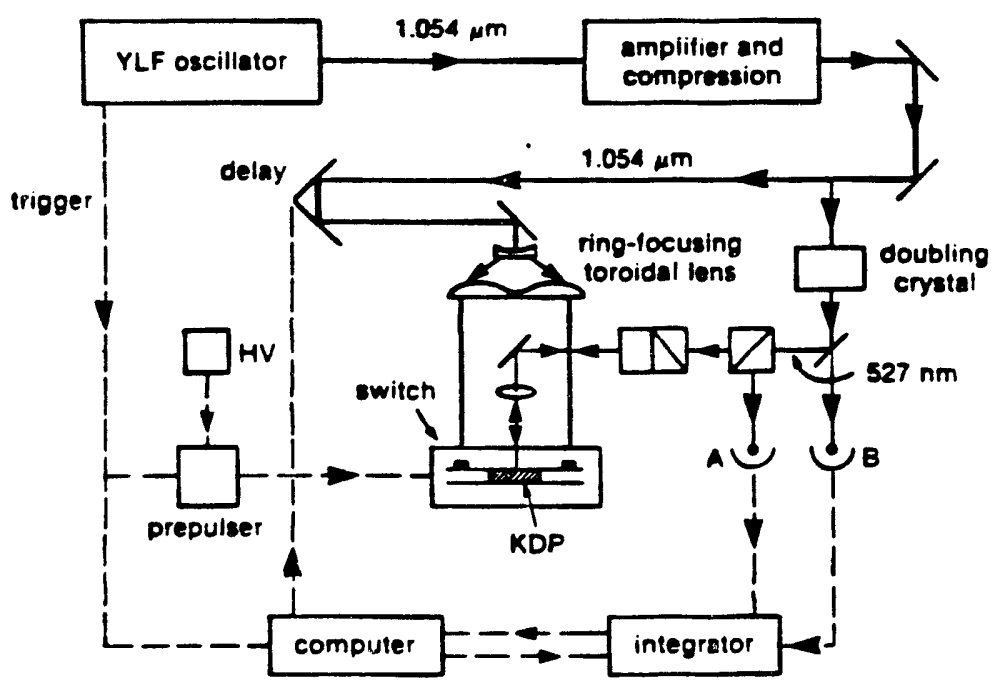

2366

Figure 4: Electro-optic sampling setup. Light from the laser is split into a switch pulse and a probe pulse. The switching pulse is focussed into a ring on the circumference of the radial transmission line with a toroidal lens. The probe pulse is used to measure the electro-optic effect in $K \mathrm{~K}^{*} \mathrm{P}$ and hence determine the voltage at the center of the structure. 


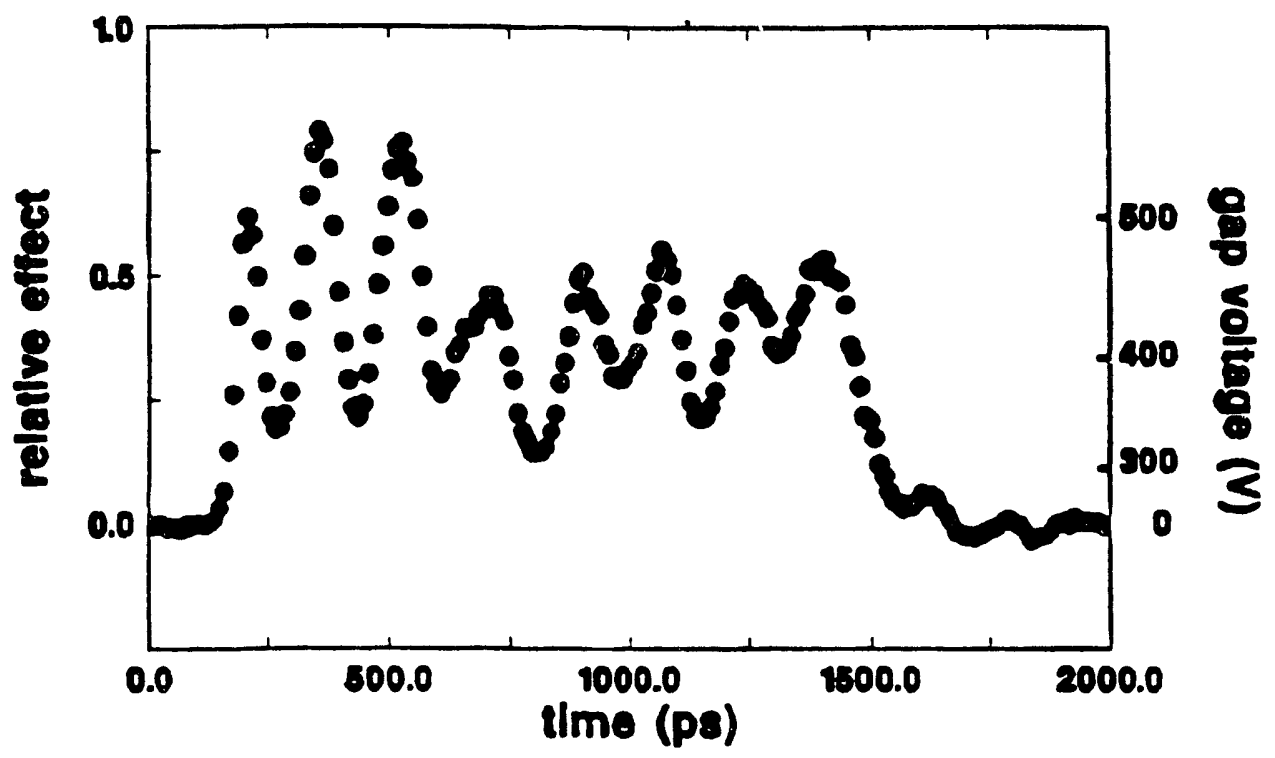

Figure 5: Electro-optic sampling curve. Time evolution of the electro-optic effect measured in $\mathrm{KD}^{*} \mathrm{P}$ crystal at the center of the radial transmission line. The voltage applied was 500 volts. Ringing in the structure is apparent.

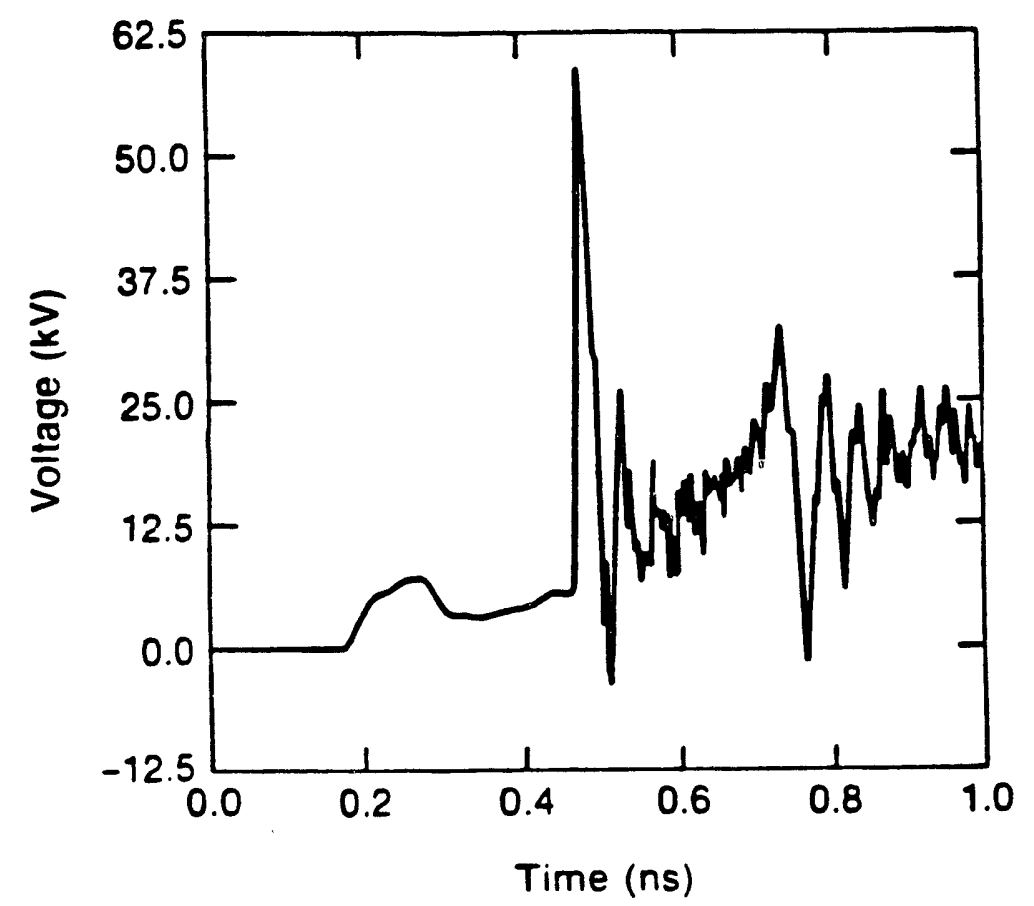

Figure 6: Model of the radial transmission line. The waveform at the center of the structure is calculated for a 0.5 picosecond closing of the switch. The voltage applied was $10 \mathrm{kV}$; the outer radius was $3.6 \mathrm{~cm}$. 


\section{(c) Electron Acceleration}

We have accelerated short electron pulses produced by photoionization. The accelerating structure is the radial transmission line discussed previously, the entire system being shown schematically in Figs. 7a,b. The structure and the electron beam analyzing and detection system are in a vacuum box maintained at $10^{-6}$ torr. The ring-focussed IR beam enters through the large front window and is incident on the semirsnductor wafer. The $U V$ beam, derived from quadrupling the IR, enters from the rea: through a quartz window and is directed onto the small beam hole in the center of the disk. The beam hole is $0.5 \mathrm{~mm}$ in diameter whereas the beam is focussed such that $\sigma \sim 100 \mu \mathrm{m}$ and impinges on the gold coating of the wafer where electrons are photoemitied.

During operation the outer ring of the semiconductor is pulsed with negative high voltage; the aluminum disk is at ground. In spite of the applied pulse the conducting surface of the wafer is kept at ground and only the outer ring becomes charged. When the IR arrives it switches the voltage of the outer ring onto the periphery of the inner surface. The pulse then propagates to the center where any electrons present are accelerated and exit through the beam hole. An external photoconductive switch is used to discharge the applied voltage 2 ns after the main pulse. This prevents the switch from going into the "lock-on" regime where the switch is most likely to be damaged. The input impedance of our structure is less than $1 \Omega$. At an applied voltage of $1 \mathrm{kV}$ the switch must therefore handle a peak power of order of MW. Our photoconductive switch performed well over $10^{6}$ shots.

It is interesting to consider the waveform of the pulsed voltage at the outer ring of the wafer as shown in Figs. 8. In the case of a $S i$ disk the switch remains closed and current continues to flow from the supply leading to the voltage droj, observed in Fig. 8(a) long after the arrival of the IR. In contrast, for $\mathrm{GaAs}$ (provided the voltage is below the lock-on value) the switch quickly opens so that there is only instantaneous drain from the supply as shown in Fig. 8(b). The gain of the structure is sensitive to good alignment and symmetric illumination of the switch by the IR. We have found that the electron energy is directly proportional to the fraction of the switch that is illuminated. Most imporiantly, if any prepulses are present the charge on the outer ring is switched before the arrival of the main pulse; this results in a uniform loading of the disk surface which prevents the propagation and amplification of the main pulse, resulting in a gain of order unity. To overcome this difficulty we used the saturable absorber described previously and carefully tuned the compression gratings.

The electrons are focussed by a solenoid coil onto a multichannel plate (MCF) located 
at the end of the biox and held at ground potential. A phosphor screen and image intensifier allow direct viewing of the electron beam. To analyze the electron energy we use a retarding grid in front of the MCP and also a small deflecting electromagnet.

To investigate the electric field at the center of the structure and the acceleration process we adjust the delay between the $U V$ and IR pulses, and measure the electron energy. Such a sampling curve is shown in Fig. 9; it was obtained with the following parameters.

$\begin{array}{ll}\text { Disk spacing } & 0.5 \mathrm{~mm} \\ \text { Beam hole } & 0.5 \mathrm{~mm} \\ \text { Characteristic input impedance } & 0.64 \Omega \\ \text { Pulse voltage } & 750 \mathrm{~V} \\ \text { Optical pulse } & \\ \quad \text { length } & 2.0 \mathrm{ps} \\ \quad \text { IR energy } & 5 \mathrm{~mJ} \\ \quad \text { UV energy } & 25 \mu \mathrm{J} \\ \text { Electron energy (peak) } & 2.6 \mathrm{keV} \\ \text { Gain } & 3.4 \\ \text { Switched current } & 1170 \mathrm{~A} \\ \text { Electrons/pulse } & 10^{\circ} \\ \text { Repetition rate } & 0.4 \mathrm{~Hz} \\ \text { Switch material }(R=3 \mathrm{~cm}) & \mathrm{GaAs}(500 \mu \mathrm{m} \text { thick) } \\ \text { Coating } & \mathrm{Au} \text { with ITO }\end{array}$

The time of arrival of the electric pulse at the center of the structure can be easily observed, as well as two repeated reflections. The spacing between reflections is $250 \mathrm{ps,} \mathrm{slightly} \mathrm{larger}$ than expected from the radius of the structure $(R=3 \mathrm{~cm})$. The electric pulse width can be estimated to be of order 10 ps. It is evident that electrons that are photoproduced before the arrival of the pulse are still accelerated. This implies that the photoproduced electrons (even at zero bias) form a plasma on the surface before recombining; the lifetime of this plasma is longer than 100 ps.

To measure the electron current we use an MCP who's output is viewed on a scope; the charge accelerated per pulse is of order $200 \mathrm{fC}$. The maximum pulsed input voltage that could be used so far was $3.2 \mathrm{kV}$ and resulting in $11.4 \mathrm{keV}$ electrons. The electron current as a function of time is shown in Fig. 10. Again the primary and reflected pulses are clearly observed. The width of these peaks is due to the fairly long lifetime of the photoproduced electron plasma which s:ales as the square root of the gas pacing. 

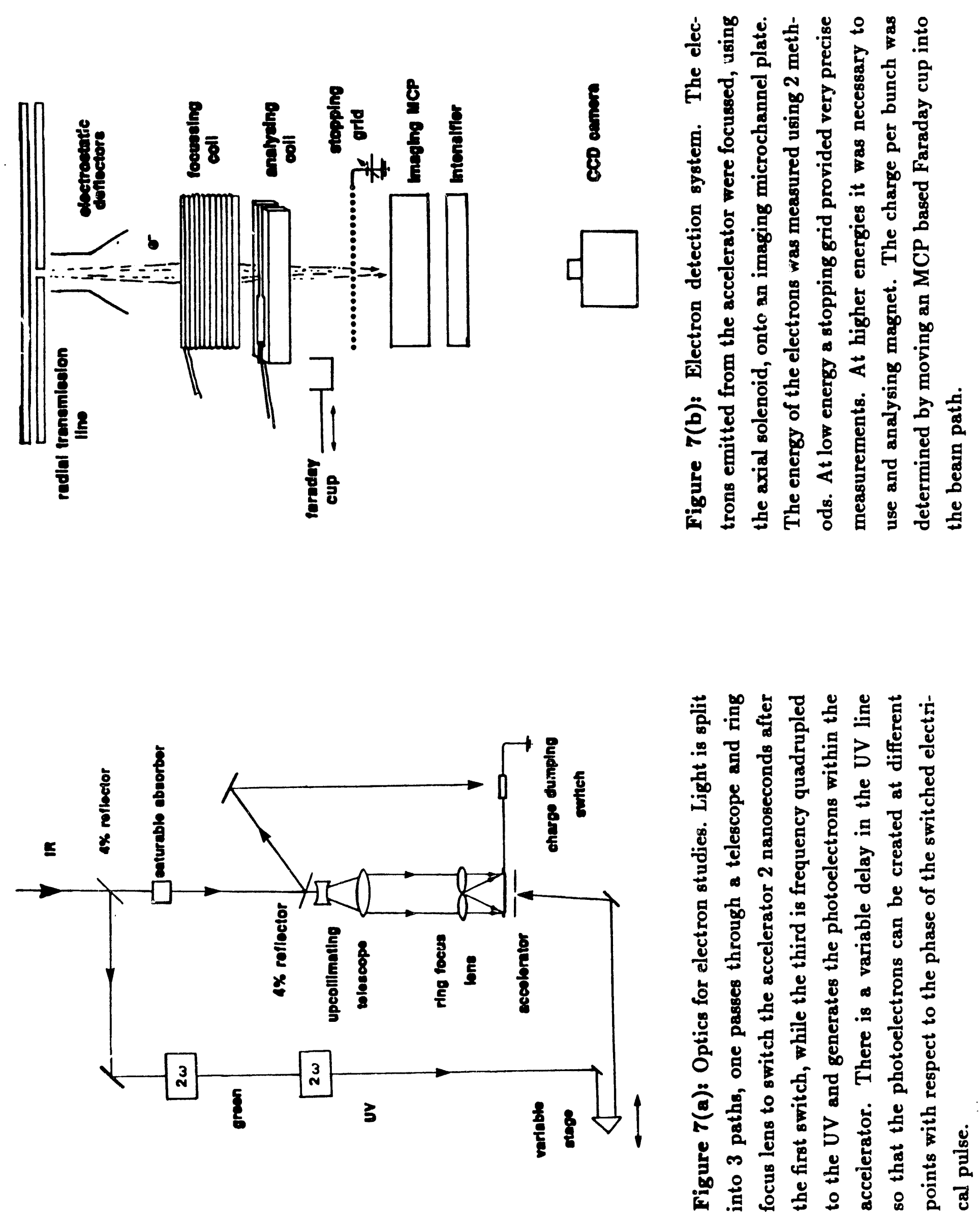


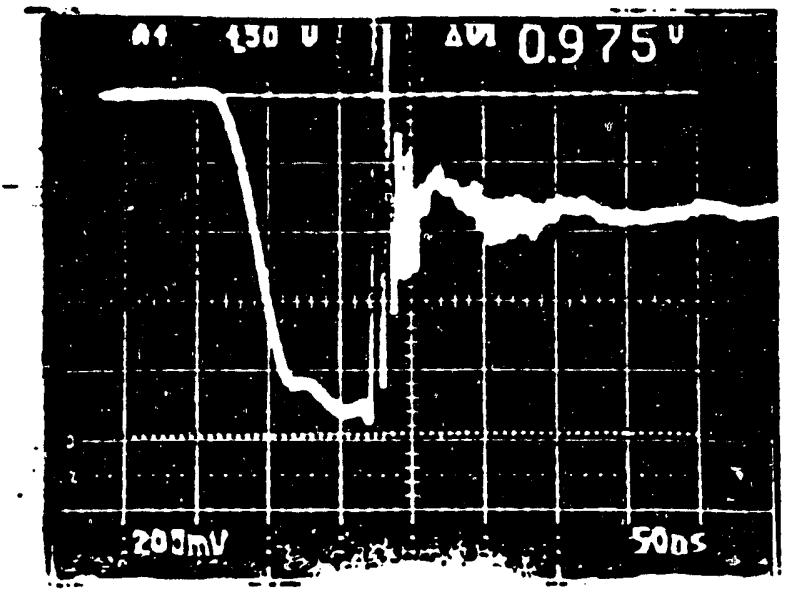

-

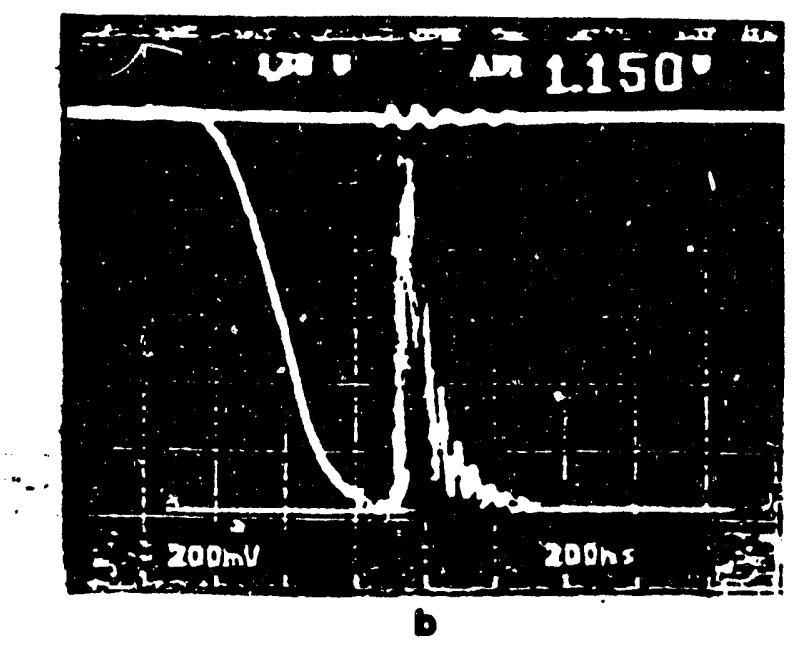

b

Figure 8: Operation of photoconductive switches. The measurements were taken on the high voltage feed side of the switch.

a. Silicon: the switch closes and remains conducting

b. GaAs: The switch closes but reopens a short time later.

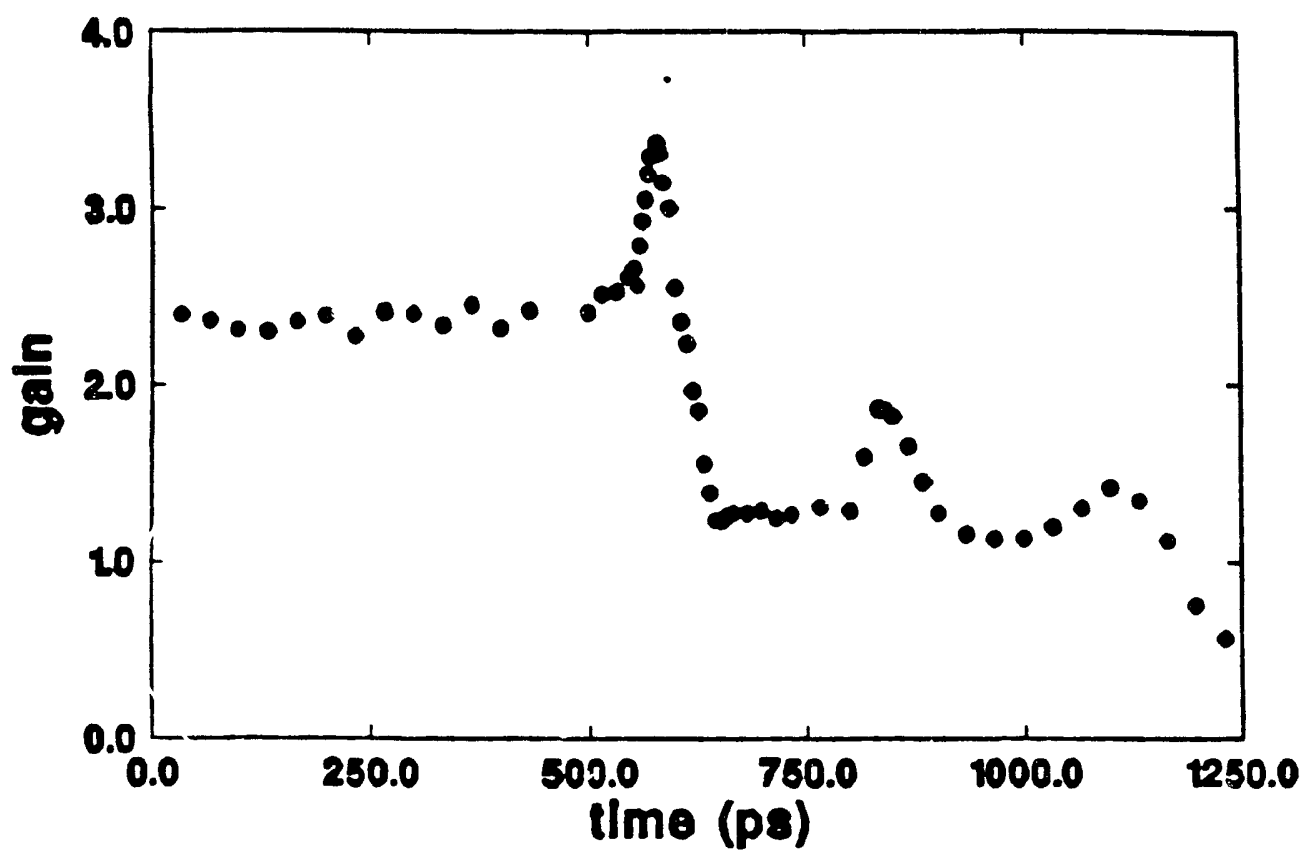

Figure 9: Electron energy gain measurement. The gain is the ratio of the final electron energy (in eV) to the applied voltage. This is measured as a function of the delay between the UV (photoelectron producing) pulse and the IR (electric field switching) pulse. Gains of this magnitude were only observed when a saturable absorber was employed. 


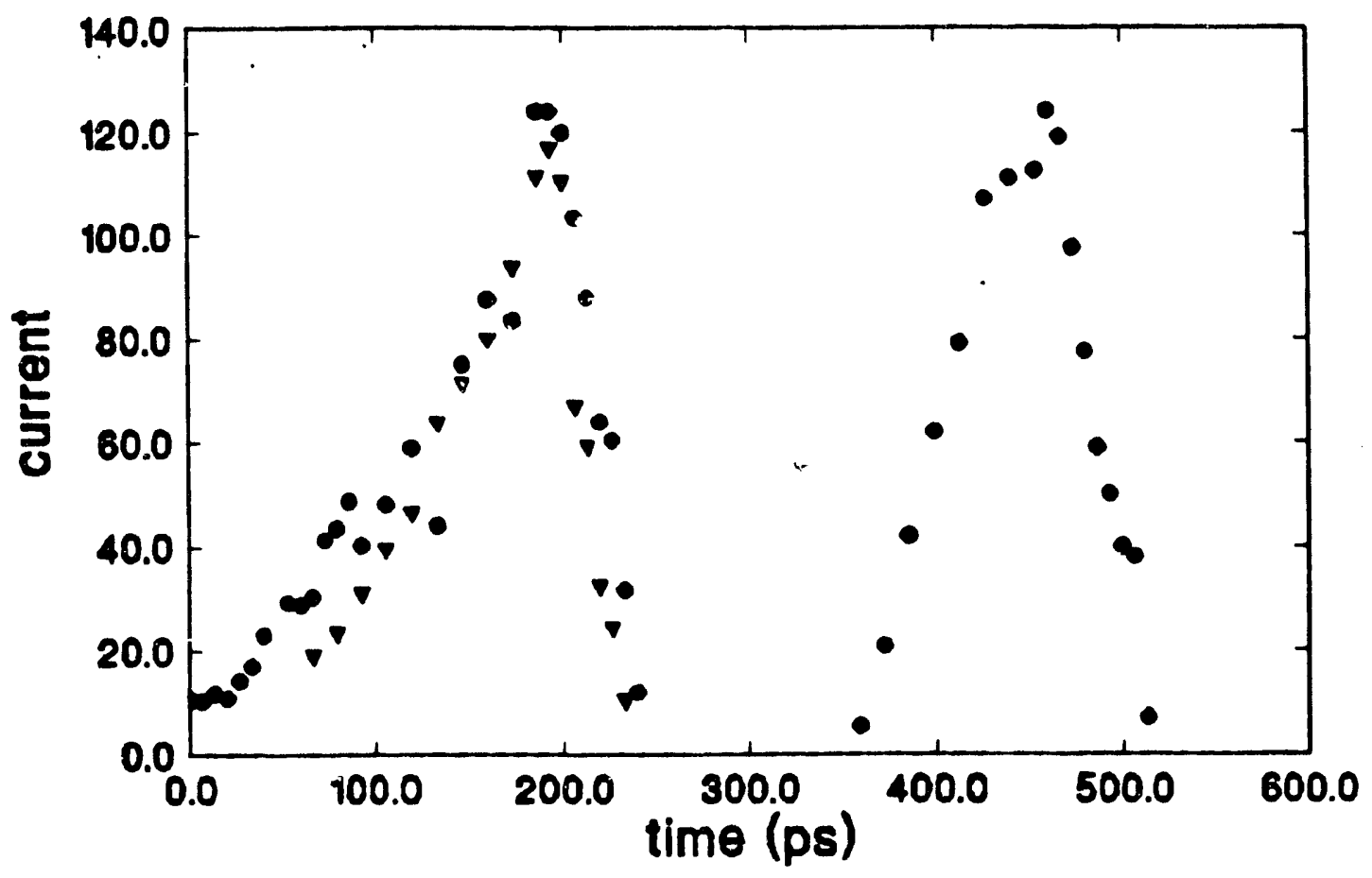

Figure 10: Electron current measurement. The electron yield as a function of the delay between the UV (photoelectron producing) pulse sad the IR (electric field switching) pulse.

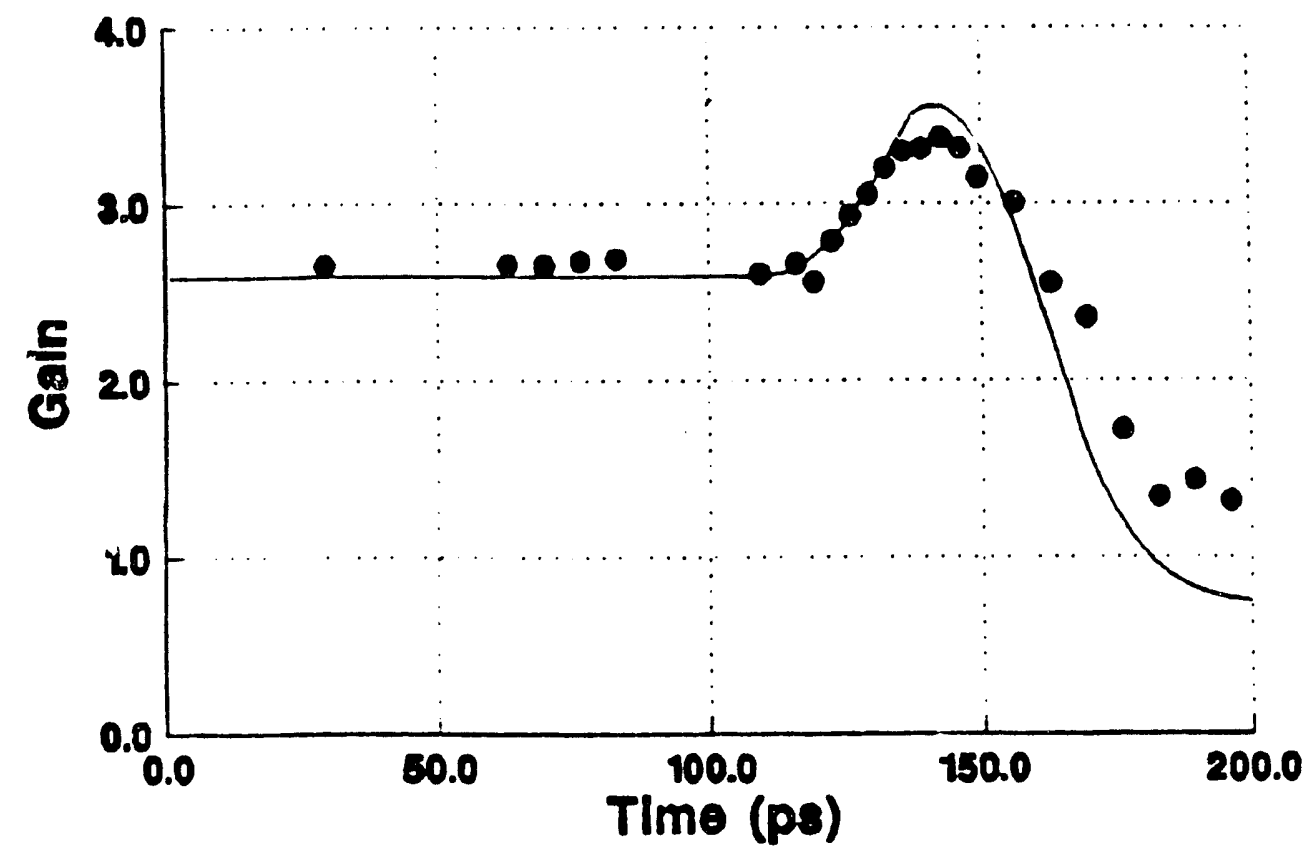

Figure 11: Modelling of electron acceleration. The solid dots are measured electron energies. The curve is the result of a modelling program. The model calculates the electric field at the center of a radial transmission line and uses this field to propogate the eleitrons across the gap. The model parameters are:

Vcitage applied

RTL radius

RTL gap
750 volts

$3.1 \mathrm{~cm}$

$0.4 \mathrm{~mm}$
Switch risetime

Switch efficiency
$38 \mathrm{ps}(10-90 \%)$

$90 \%$ 
The acceleration process has been modeled by taking into account the time dependence of the compressed electric field pulse and the time at which the electrons are photoemitted. The electrons are then stepped through the accelerating gap (1-dimensional integration in time). It is assumed that once the electrons are produced, they are available for acceleration at any later time when the electric field is present. The results are shown in Fig. 11 and do not include the reflected pulse. The qualitative agreement with the data supports the conclusion that the electrons are accelerated by the compressed pulse.

In conclusion we have demonstrated that short electron pulses can be accelerated by pulsed power using a radial compression technique. The results obtained are in qualitative agreement with the theoretical predictions. For a realistic accelerator the pulsed HV holdoff as well as the electron yield must be increased. With these improvements the present structure would be an electron source of high brilliance.

\section{(d) Future Plans}

We would like to continue this work with the main objective of increasing the voltage that can be held off by the structure. One approach is to use a linear switch to launch a high voltage pulse into the acceleration region. Our goal would be to reach $25 \mathrm{kV}$ across a $0.25 \mathrm{~mm}$ gap. This simpler geometry is much more easy to replicate and we will renew our effort of finding a suitable design for a multicell structure.

Another possible approach is related to recent contacts with Dr. H. Ikezi of General Atomics (San Diego). His group has developed a dielectric pulse compressor delivering $70 \mathrm{kV}$ in $1 \mathrm{nsec}$. [H. Ikezi et a., J. Appl. Phys. 64, 6836 (1988)]. If this device could be combined with our photoconductive switching radial compression technique one could expect to generate ultrashort electrical pulses approaching a MV.

\section{iii. The Superconducting Laser RF Gun}

As mentioned in the program overview we are presently concentrating our effort on realizing a high brilliance electron source in collaboration with Cornell (Drs. L. Haud and H. Padamsee). The source is based on a superconducting cavity in which the electrons are directly produced by photoionization using our short pulse laser. There have been proposals for such an r.f. gun in the literature but we know of no practical realization as yet.

To obtain a high accelerating field at the emission point we may use a "mushroom" cavity, namely a design where a dimple is introduced at the center of the flat surface. This is shown in Fig. 12 where we also indicate the field lines. The calculated gradient at the dimple is of order of $300 \mathrm{MV} / \mathrm{m}$. The cavity shown operates at $6 \mathrm{GHz}$ and is made of Niobium. 


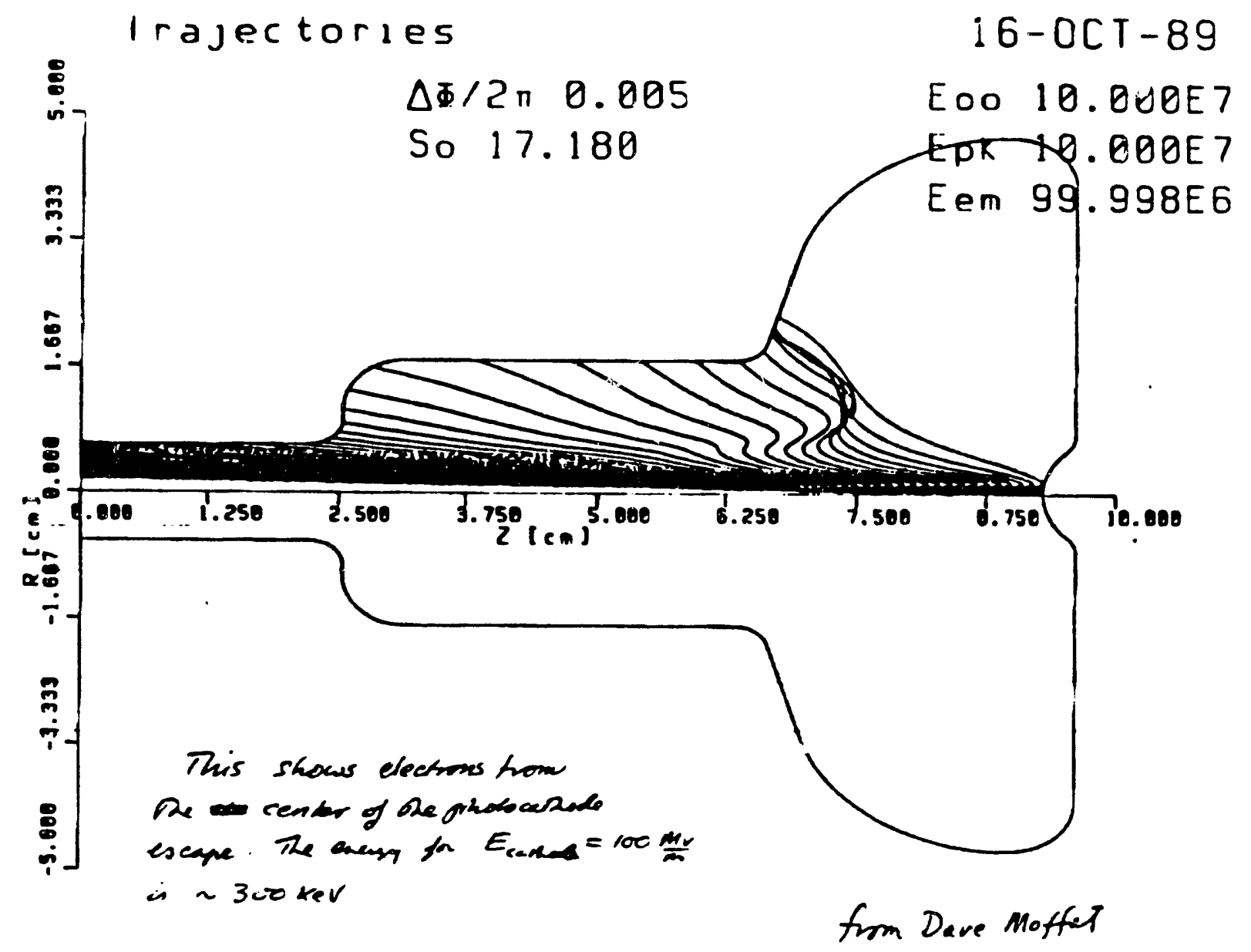

Figure 12: Outline of the superconducting cavity and of expected electron trajectories. 


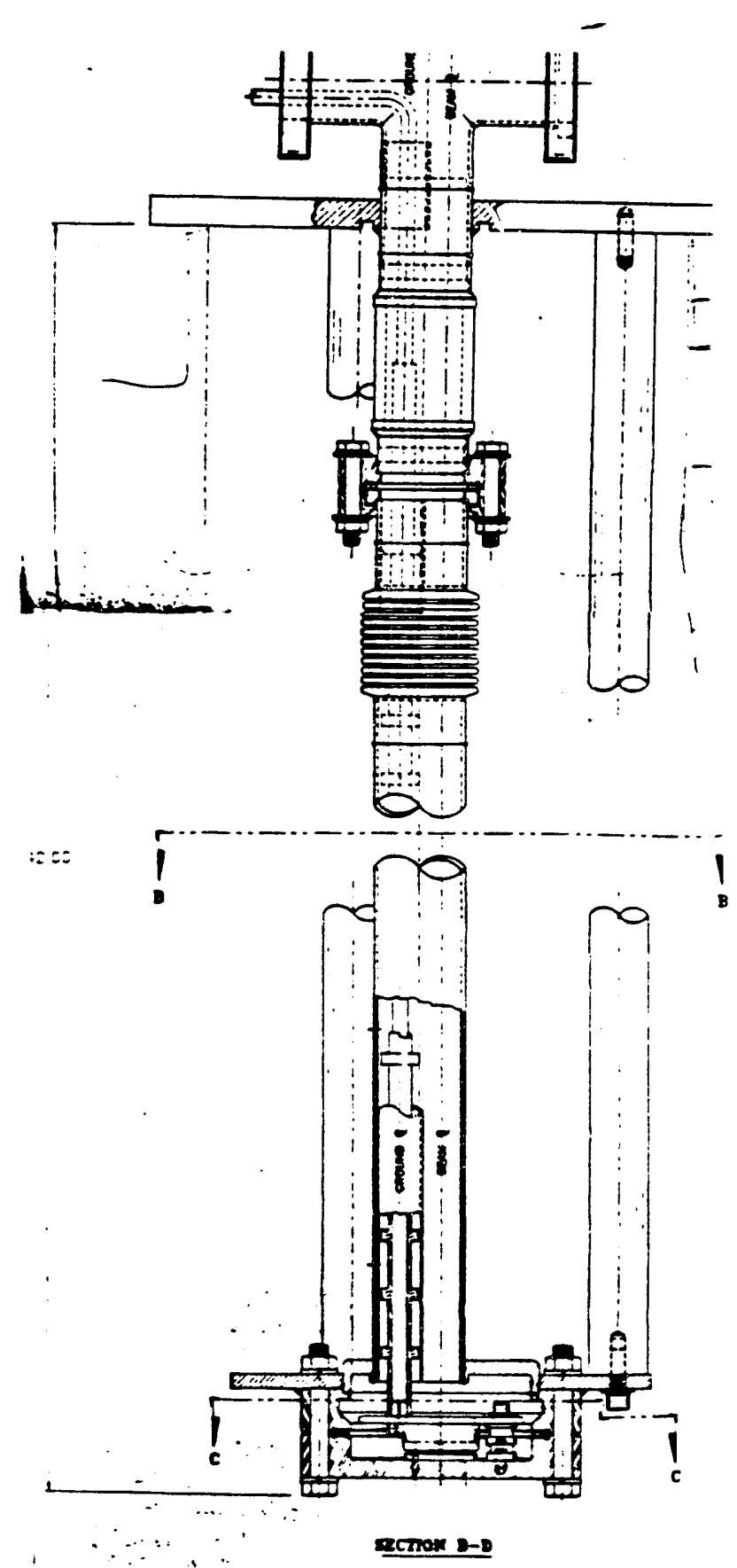

Figure 13: Cryostat to be used for photoemission studies from superconducting Niobium. 
The laser pulse will be directed onto the cathode through the beam line aperture and a transition section will be attached to the eryogenics so that the electron beam diagnostics can be carried out at room temperature. Operation of the electron gun involves the necessary synchronization of the laser oscillator with the phase locked cavity microwave source. This can be achieved by a proper feedback circuit. If beam focussing becomes necessary we will provide a solenoid coil on the neck of the cavity. We hope to achieve a photoemission efficiency of $\sim 10^{-4}$ (a factor of 100 better than at present) in order to keep the laser power on the cathode low, less than $1 \mu \mathrm{J}$ per pulse.

The design goals for the source are as follows:

\section{Energy}

Normalized emittance

Energy spread

Electrons/pulse

Pulse length

$$
\begin{aligned}
& >5 \mathrm{MeV} \\
& <10^{-6} \mathrm{~m}-\mathrm{rad} \\
& <0.01 \\
& 10^{\circ} \\
& 20 \mathrm{ps}
\end{aligned}
$$

Our plan is to first test $\mathrm{Nb}$ cathodes at room and at liquid helium temperatures. To this effect we heve constructed a cryostat (Fig. 13) with which we will conduct tests during the summer. The geometry is similar to that of the microwave cavity so that all the laser illumination problems can be solved using this prototype. A cryostat to hold the cavity is under construction at Cornell but eventually will have to be transported to Rochester where the tests will be carried out. For this reason we need to acquire some $6 \mathrm{GHz}$ microwave equipment for the operation of the cavity in Rochester, as detailed in our equipment request.

We expect to have preliminary results from this effort by the end of the year. The question of beam diagnostics at $5 \mathrm{MeV}$ is now being addressed and will be based on a magnetic spectrometer.

\section{iv. A Possible Picosecond X-ray Source}

We propose to scatter a short UV pulse from our laser system, off a $5 \mathrm{MeV}$ electron beam. The electron beam will be obtained from the superconducting laser driven source discussed in the previous section. The energy of the backscattered photons is given by

$$
\omega=\frac{4 \gamma^{2} \omega_{0}}{1+2 \gamma^{2}(1-\cos \theta)}
$$

where $\omega_{0}$ is the incident photon energy, $\gamma=E / m$ for the electrons, and $\theta$ the laboratory scattering angle ( $\theta=0$ corresponds to backscattering). For $5 \mathrm{MeV}$ electrons and $\omega_{0}{ }^{\circ}=3.5$ 
Compton Scattering

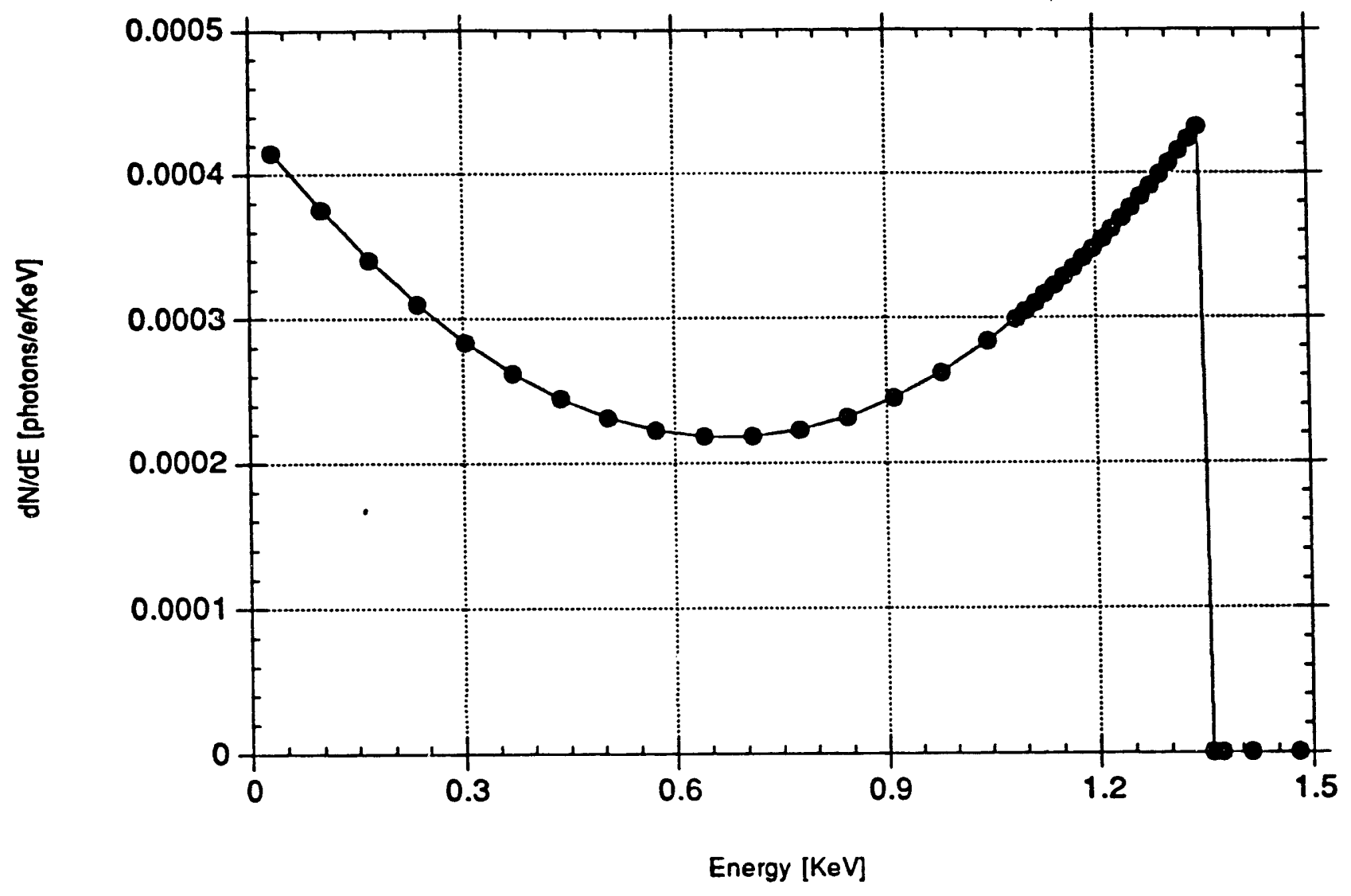

Figure 14: X-ray spectrum from backscattering a UV pulse from $5 \mathrm{MeV}$ electrons.

\begin{tabular}{|r|r|}
\hline Electron parameters: & \\
\hline Energy: & $5.00 \mathrm{MeV}$ \\
\hline Bunch length (FWHM): & $10.00 \mathrm{psec}(0.300 \mathrm{~cm})$ \\
\hline Radial sigma: & 100.00 microns \\
\hline Laser parameters: & \\
\hline Wavelength (energy): & 0.35 microns (3.542 eV) \\
\hline Pulse energy: & 0.3000 Joules \\
\hline FWHM of pulse: & 1.00 psec \\
\hline Tau of pulse: & 0.57 psec \\
\hline Peak power: & 0.2644 teraWatts \\
\hline FD of focussing element: & 100.00 \\
\hline Maximum Eta: & 0.0331 \\
\hline Peak intensity: & $2.44 e+16$ Watts/cm² \\
\hline Peak photon flux: & $4.30 e+34 \# / \mathrm{cm}^{\wedge} 2 / \mathrm{sec}$ \\
\hline Sigma of beam waist: & 15.75 microns \\
\hline Rayleigh range: & 4454.99 microns \\
\hline Tolal rate $=$ & $3.9303 \mathrm{e}-04$ photons/e \\
\hline Klein-Nishina $=$ & $3.9285 \mathrm{e}-04$ photons/e \\
\hline
\end{tabular}


of Mr. T. Blalock who has greatly contributed to laser development and diagnostics. The experimental equipment is located at the Laboratory for Laser Energetics and benefits from the mutual interaction with the other groups involved in high power, pulsed laser work in residence at the LLE.

Presentations of this work were made at several laboratories and universities during the past year: Dr. A. Melissinos gave a seminar at CERN; Dr. L. Kinglsey presented a talk at the Conference on Lasers and Electro-Optics at Annaheim CA in May 1990, and at the CLEO Meeting in Boston in December. Dr. E. Lincke presented a poster at the 2nd European Conference on Particle Accelerations in Nice in June 1990; he also gave seminars at the SSC Laboratory, at BNL/NSLS and at IBM/Fishkill Research Labs. 


\section{Publications}

Because the Laser Switched Linac project is nearing completion we give below a comprehensive list of all publications up to the present (1988-1990).

1. C. Bamber, W. Donaldson, T. Juhasz, L. Kingsley, and A. C. Melissinos "Radial Compression of Picosecond Electrical Pulses"

Particle Accelerators, $\underline{\text { 23, }} 255$ (1988).

2. E. Lincke

"Solid State Switching"

Proceedings of the Switched Power Workshop, Shelter Island, NY, BNL-52211, p. $12-43$; p. 243-247 (1988).

3. C. Bamber, W. Donaldson, T. Juhasz, L. Kingsley, E. Lincke, and A. C. Melissinos "Radial Compression of Picosecond Electrical Pulses"

Proceedings of European Particle Accelerator Conference, Rome, June 1988, World Scientific Publishing, S. Tazzari ed., (1989), p. 1267.

4. L. E. Kingsley and W. R. Donaldson

"Electro-Optic Surface Field Imaging System"

Digest of Technical Papers, 7th IEEE Pulsed Power Conf., R. White and B. H. Bernstein, eds., (IEEE, NY) 1989, p. 376-379.

5. C. Bamber "Laser Switched Power Linac"

AIP Conference Proceelings 193, Advanced Accelerator Concepts, C. Joshi, ed., 1989, p. 1.

6. W. R. Donaldson and L. E. Kingsley

"Electro-Optic Imaging of the Internal Fields of a GaAs Photoconductive Switch"

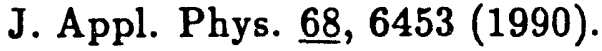

7. L. E. Kingsley and W. R. Donaldson

"Electro-Optic Imaging of Surface Electric Fields in High-Power Photoconductive Switches"

IEEE Transactions on Electron Devices $\underline{37}, 2449$ (1990).

8. E. Lincke

"Acceleration of Picosecond Electron Bunches in a Radial Transmission Line" to appear in Proceedings of the European Accelerator Conference, Nice, France, June 1990.

9. L. E. Kingsley

"A Study of Picosecond High-Voltage Photoconductive Switching Using Electrooptic Diagnostics and Computer-Aided Analysis"

$\mathrm{Ph} . \mathrm{D}$. thesis, University of Rochester, January 1991, Laboratory of Laser Energetics Report No. 220. 

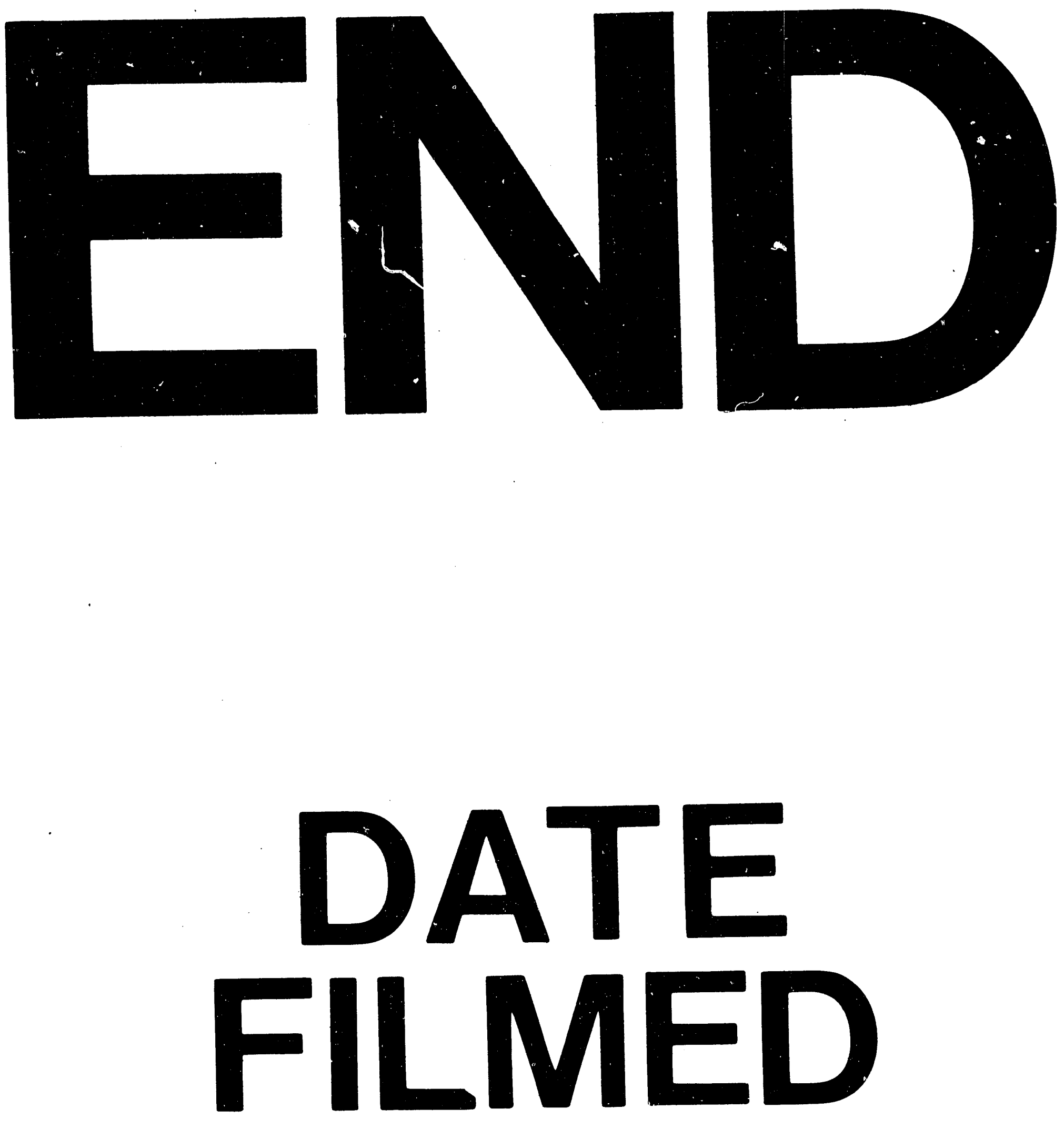

1

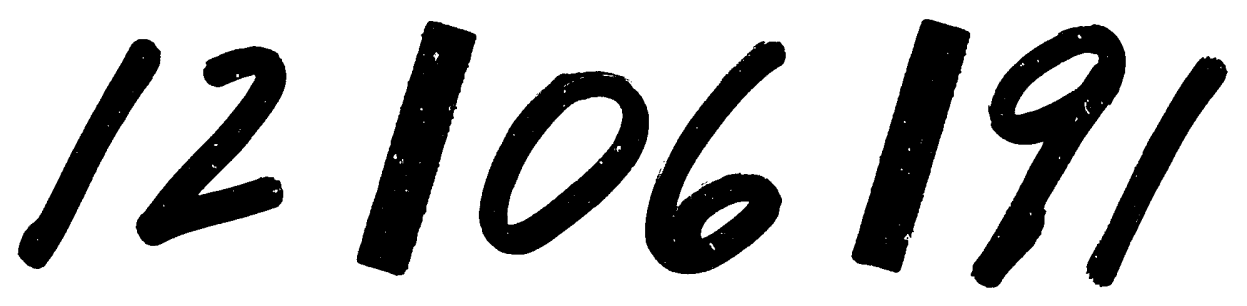

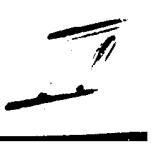


